\title{
Joint meeting of the Association of British Neurologists and the Norwegian Neurological Association on the coastal steamer Hurtigruten, 6-9 May 2001
}

\author{
Mitochondrial myopathies \\ L Bindoff Department of Neurology, Haukeland Hospital, University of Bergen, \\ Norway
}

Mitochondrial respiratory chain dysfunction is a potent cause of disease. Where once it was the realm of the interested neurologist only, mitochondrial disease is now common and may present to any and indeed all of the medical and surgical specialties.

The term mitochondrial myopathy is used as the descriptive term for this group of disorders where muscle disease is an important manifestation, albeit one that is rarely found in isolation. Classic phenotypes include Kearns-Sayre syndrome, chronic progressive external ophthalmoplegia, MELAS, MERRF, and Leber's optic atrophy. These disorders result from mutations of the mitochondrial genome (mtDNA), which encodes 13 respiratory chain proteins. More recently, genetic defects involving proteins not encoded by mtDNA have also been identified-for example, in Friedreich's ataxia, one form of hereditary spastic paraplegia, and an infantile cardiomyopathy.

The range, diagnosis, and investigation of mitochondrial disease, with particular reference to neurological manifestations, are reviewed.

\section{Inflammatory myopathies}

D Hilton-Jones Oxford MDC Muscle and Nerve Centre, Oxford, UK; Radcliffe Infirmary, Oxford, UK

Introduction: The classification of this rather disparate group of conditions is reviewed. The commonest are the idiopathic inflammatory myopathies, comprising polymyositis (PM), dermatomyositis (DM), and inclusion body myositis (IBM). They enter into the differential diagnosis of other neuromuscular conditions and incorrect or late diagnosis is common. This may have two consequences. PM and DM are treatable conditions and failure to recognise them may deny the patient effective therapy. Conversely, misinterpretation of pathological changes in other disorders - notably, some forms of limb girdle muscular dystrophy - may result in inappropriate and damaging steroid therapy. Inclusion body myositis is unresponsive to treatment and probably has a different pathogenesis to PM and DM and it is described separately Clinical features (PM and DM): Proximal weakness is always greater than distal, but the highly selective involvement of specific muscles characteristic of the muscular dystrophies is not seen. The onset may be acute (in DM) or chronic, sometimes over years (in PM). Except in acute cases it is usually painless, but exercise induced pain may be seen in DM. Dysphagia is commoner in DM and most often seen in acute or severe cases. Raynaud's phenomenon is seen in DM. Both may be accompanied by interstitial lung disease, associated with the presence of the antibody anti-Jo1. Skin changes in DM may be subtle or absent. Typical changes include erythema over sun exposed areas (face and upper chest) and the knuckles, dilatation of nail bed capillaries and, less commonly than many textbooks suggest, violaceous discolouration of the eyelids. DM is associated with carcinoma in about $20 \%$ of cases.

Diagnosis: The gold standard is muscle biopsy. The characteristic changes are reviewed, with comments relating to how such changes have led to the conclusion that, despite the clinical similarities, these two conditions each have a different immunopathogenic basis.

Treatment: Despite recent insight into immunopathogenic mechanisms, treatment remains a rather blunderbuss approach to immunosuppression with prednisolone and other immunosuppressant drugs. Therapeutic choice, in the absence of randomised trials, is based upon expert opinion.

Inclusion body myositis: This is the commonest acquired myopathy in the elderly. Many patients previously labelled as having steroid resistant polymyositis, or isolated quadriceps myopathy had this condition. The most characteristic presentation is with wasting and weakness of quadriceps accompanied by specific weakness of the finger flexors. The muscle involvement is often asymmetric. Dysphagia may be an early feature. The characteristic muscle biopsy findings are reviewed. Immunosuppressant therapy rarely confers significant benefit and it is arguable whether it should be attempted.

A new non-dystrophic painful myotonia with dominant inheritance

S Løseth, T Torbergsen, $\mathrm{Nj}$ Brautaset, E Stålberg Departments of Neurology, Tromsø University Hospital and Vestfold County Hospital, Norway; Department of Clinical Neurophysiology, Uppsala University Hospital, Sweden

We report a variety of painful non-dystrophic myotonia not previously described. The hereditary non-dystrophic myotonias are caused by mutations in the gene coding for the chloride or sodium ion channels in the skeletal muscle cell membrane. In the chloride channel disorders the myotonic symptoms are most pronounced when initiating movements after rest, whereas in sodium channel myotonia the symptoms usually appear and increase during exercise. Pain is a rare symptom in myotonia. We have found an unusual myotonic disorder in a 34 year old woman and her 14 year old daughter. Painful cramps occur during and after exercise in the mother, and both patients demonstrate unusual muscle contractions in the tongue. The electromyographic recordings reveal positive waves, fibrillation potentials, myotonic discharges, and extraordinary findings consisting of trains of high frequency positive potentials with very high amplitudes. In the present study we try to evaluate the generator for these potentials. It is likely that these discharges represent activity from bundles of neighbouring muscle fibres activated by ephaptic transmission.

A novel autosomal dominant inclusion body myopathy with early respiratory failure links to chromosome $6 \mathrm{q}$

PF Chinnery, A Dearlove, MA Johnson, TJ Walls, GJ Gibson, PRW Fawcett, S Jamieson, JJ Fulthorpe, M Cullen, P Hudgson, KMD Bushby University of Newcastle upon Tyne, Newcastle, UK

We carried out a standardised clinical assessment and detailed respiratory function tests on 24 individuals from a three generation family with a novel autosomal dominant distal myopathy.

The myopathy presented in mid-adult life with tibialis anterior weakness. Lung function tests revealed progressive global respiratory muscle weakness detectable from the time of presentation, with preferential diaphragmatic involvement in some cases.

There was a striking correlation between the clinical and pathological features. Clinically unaffected muscles had minimal pathological change. Fibre splitting, eosinophilic inclusions, and vacuoles with basophilic rims were seen in moderately affected muscles, and fat and fibrous connective tissue replaced muscle fibres in the severely involved muscles. The inclusions were congophilic and reacted with antibodies to desmin, $\beta$-amyloid, and phosphorylated tau.

A genome-wide linkage study was carried out using 350 microsatellite markers. Multi-point analysis localised the causative gene to a $5 \mathrm{cM}$ region on chromosome $6 \mathrm{q}$ with a maximum LOD score $>3.5$.

In conclusion, the family have a unique disorder that is both phenotypically and genetically distinct. It is likely that this disease is caused by mutations in a gene on chromosome $6 \mathrm{q}$ that is essential for respiratory muscle function.

The clinical usefulness of EEG for the diagnosis of alcohol related epileptic seizures

G Bråthen, T Sand, R-P Michler, E Brodtkorb, G Helde, G Bovim Department of Clinical Neurosciences, Faculty of Medicine, Norwegian University of Science and Technology, Trondheim, Norway 
The aim of this study was to investigate whether EEG could discriminate between alcohol related seizures (ARS) and seizures unrelated to alcohol use.

ARS were defined as seizures in patients with scores $\geqslant 8$ in the alcohol use disorders identification test (AUDIT). Twenty two patients with ARS and no known epilepsy (ARSwE), 21 AUDIT negative epilepsy patients with seizures (ES), and 30 other AUDIT negative patients with seizures (OS) were studied. Thirty seven epilepsy outpatients and 79 sciatica inpatients were used as controls.

Epileptiform and slow activity were less frequent in the ARSwE than the ES group. Alpha amplitude was lower in the ARSwE than the other groups. Photoparoxysmal activity was not seen. EEG was associated with a larger negative predictive value (78\% probability of nonARS if EEG was abnormal) than a positive predictive value (55\% probability of ARS if EEG was normal).

A definitely abnormal EEG suggests epilepsy or symptomatic seizures unrelated to alcohol. The predictive value of a normal EEG is limited, but the typical postictal finding in ARS is nevertheless a normal low amplitude EEG record.

Study of the neurological, cognitive, and behavioural features of spinocerebellar ataxia type 8

A Zeman, J Stone, M Porteous, L Barron, J Warner Department of Clinical Neurosciences, University of Edinburgh, Western General Hospital, Edinburgh, $U K$

Spinocerebellar ataxia type 8 (SCA 8) is a recently described disorder due to an expansion of non-coding CTG repeats on chromosome $13 q 21$, but its validity is in doubt. Analysis of samples from 100 patients with undiagnosed ataxia in our laboratory revealed four with unequivocal expansions versus one expansion among 2380 chromosomes from anonymised controls.

We have previously described a patient with the expansion who presented with features of frontal lobe dysfunction in addition to a cerebellar syndrome; his mother had a similar genotype and phenotype. We therefore assessed this initial case and the four additional subjects, using clinical methods and psychiatric and neuropsychological tests to establish whether the expansion associates with a consistent phenotype, and, if so, whether it has a neuropsychiatric dimension.

Our patients had combined CTA/CTG repeat lengths of 131 to more than 170 . Symptoms first occurred between the ages of 14 and 47 , mean 32. Neurological features included a cerebellar syndrome in $4 / 5$, upper motor neuron signs in $3 / 5$, and sensory disturbance in $2 / 5$. Neuroimaging revealed cerebellar atrophy in $4 / 5$. Mini mental state examination (MMSE) was normal in all but two patients who had a dysexecutive syndrome with obsessive-compulsive disorder in a third. The SCA 8 expansion is probably pathogenic; its phenotype includes cognitive and behavioural features.

\section{Treatment of autoimmune neuropathy}

M Donaghy Department of Clinical Neurology, University of Oxford, UK

Acute idiopathic polyneuritis (Guillain-Barré syndrome) occurs in two main forms: motor-sensory demyelinating and acute motor axonal. Spontaneous recovery occurs in both conditions, and general medical care is an important determinant of survival: ventilation and intubation, prophylaxis against thromboembolism, treatment of cardiac arrythmia, nutrition, and treatment of infection. Plasma exchange (PE) hastens improvement and recovery of walking, and reduces ventilation time. High dose intravenous immunoglobulin (IvIg) is similarly effective to PE but more convenient, and subgroup analysis suggests that it may be better for the motor axonal subgroup. Despite contemporary treatment, the mortality is $4 \%-5 \%$, only $60 \%$ make a full recovery, $23 \%$ show residual abnormalities, and $17 \%$ are unable to walk at 1 year.

The two main chronic idiopathic polyneuropathies are chronic inflammatory demyelinating sensory motor polyneuropathy (CIDP) and multifocal motor neuropathy with conduction block (MFMN). Most patients with CIDP can be improved substantially with long term alternate day steroids. PE produces temporary improvement in $80 \%$ of patients, and is particularly recommended for slow responders, severe initial disability, reversal of relapse, or if steroids are contraindicated. IvIg improves about $65 \%$ of CIDP patients for between 4 and 10 weeks and should be used in similar indications as PE. Treatment efficacy should be monitored by objective measures, such as walking speeds. By contrast, MFMN often deteriorates with steroids, but responds promptly to IvIg with response lasting 6-12 weeks. Regular maintenance IvIg scheduled to avoid relapses seems to prevent background deterioration and can be administered at home. Cyclophosphamide is also effective in MFMN, but the side effect profile is unfavourable for chronic administration. It is unusual for patients with CIDP or MFMN to be able to stop therapy without relapse.

No formal trials of therapy have been conducted for vasculitic neuropathy. Prednisolone alone often provides adequate suppression of non-systemic vasculitic neuropathy. Neuropathy due to Wegner's granulomatosis or polyarteritis nodosa usually requires cyclophosphamide to suppress underlying disease activity. Cyclophosphamide should also be used in non-systemic vasculitic neuropathy if rapidly progressive and destructive. Therapy for vasculitis aims firstly to prevent further peripheral nerve damage, and secondly to suppress the vasculitis and allow axonal regeneration.

\section{Guillain-Barré syndrome, a role for IgG Fc receptors}

C Vedeler Department of Neurology, Haukeland Hospital, University of Bergen, Norway

Guillain-Barré syndrome (GBS) is an acute inflammatory demyelinating polyneuropathy that is mediated by both humoral and cellular immune components. Receptors for IgG ( $\left.F_{C} \square R\right)$ link the humoral and cellular immune responses by targeting immune complexes to effector cells. Different $F_{c} \square$ R show variability in their distribution, strength, and capacity of binding different IgG subclasses. We have found that $F_{c} \square$ RIIA, Fc $\square$ RIIIA, and Fc $\square$ RIIIB genotypes and allele frequencies were similar in 62 patients with GBS and 89 healthy controls from western Norway. However, patients homozygous for the Fc $\square$ RIIIB neutrophil antigen (NA) 1 allele had significantly less severe disease than patients heterozygous or homozygous for the NA2 allele. The Fc $\square$ RIIIB NA1/NA1 genotype has high affinity for IgG1 and IgG3, and clearance of circulating autoantibodies and immune complexes may be therefore be of importance in the pathogenesis of GBS. The results implicate $F_{C} \square$ RIIIB as a disease modifying gene in GBS.

Peripheral nerve topography as promoter for functional recovery after nerve repair

RG Hallin Division of Clinical Neurophysiology, Huddinge Hospital, Sweden; Department of Clinical Neurosciences, Royal Free Hospital School of Medicine, London, UK

The existence of a functional neural organisation by modality and somatotopy is well founded and commonly accepted in the CNS, both in subhuman species and in humans. By contrast, prevailing orthodoxy rather emphasises the lack of such an organisation in the peripheral part of the neuraxis. However, recent findings from comparative microneurography - that is, exploration of human nerves with tungsten electrodes or concentric needle electrodes under similar conditions show that nodes of Ranvier cluster in certain regions of a fascicle and that these nodes belong to fibres of the same modality which innervate the same skin area.

Bundles of afferents of common modality may serve as projection modules processing peripheral events to cognitive levels and may also act to conserve functional gnosis when peripheral nerve fibres are lost. This organisation may also be critical as a substrate to promote reinnervation after peripheral nerve suture. The segregated fibre organisation will tend to re-establish function even if some fibre misguidance occurs during outgrowth. Functional restitution would be jeopardised if a chaotic fibre organisation were prevailing in the fascicles. An ordered intraneural topography in the periphery might also be of significance for recovery after avulsion injuries treated by nerve root implantation into the spinal cord.

Thus, human nerve fibres are well organised both in the periphery and in the CNS, which possibly has profound significance both under normal conditions and in disease.

\section{Anatomical and structural changes related to carpal tunnel syndrome}

SI Bekkelund, C Pierre-Jerome University Hospitals of Tromsø; Norway; Ulleval Hospital, University of Oslo, Norway

There are many controversies regarding carpal tunnel syndrome (CTS). The relation between symptom production and structural changes in the carpal tunnel affecting the median nerve has been subject to several studies. To test the hypothesis that the size of the carpal tunnel may play a part in the pathophysiology of CTS, we performed a controlled MRI volumetric study in a group of randomly selected patients. We also studied the relation between median nerve functions 
and the size of the carpal canal in two asymptomatic groups: (a) handworkers and $(b)$ rheumatoid arthritis (RA). The size of the carpal tunnel was not significantly different in CTS patients compared with controls, but the narrowest point was located near the canal outlet. The handworkers had impaired neurophysiological variables of the median nerve whereas the volume of the canal was almost the same in both groups. In patients with RA without median nerve abnormalities, a significant larger volume of the canal was found. From these studies we conclude that the anatomical size of the carpal tunnel alone does not predict CTS. The role of the median nerve configuration and the ability of the nerve to resist pressure from adjacent tissue should be clarified.

\section{Anti-MuSK antibodies in seronegative myasthenia} gravis: a cause of denervation

J Newsom-Davis, J McConville, R Kennett, P Anslow, W Hoch, A Vincent Department of Clinical Neurology and Neurophysiology, University of Oxford, Oxford, UK

We have recently investigated the presence of $\operatorname{IgG}$ antibodies to MuSK in myasthenia gravis (MG) patients seronegative for antiacetylcholine receptor (AChR) antibodies. MuSK is a muscle specific kinase, expressed at the fetal and adult neuromuscular junction that plays a crucial part during development, initiating AChR clustering.

An enzyme linked radioimmunoassay (ELISA), with rat MuSK as antigen, detected anti-MuSK antibodies in a group of $17 / 24$ seronegative MG patients. In vitro studies showed that these antibodies bind to the extracellular domain of MuSK and strongly inhibit AChR clustering. The focus of the present report is the youngest patient in our series of anti-MuSK positive MG. She developed profound ocular and bulbar weakness at the age of 2.5 years, and less severe generalised weakness. The bulbar weakness failed to respond to prolonged prednisolone treatment. Aged 20, no electromyographic activity could be detected in the frontalis and orbicularis oculi muscles, either stimulus evoked or on volition, with only occasional very small motor units in orbicularis oris. MR imaging confirmed profound wasting of facial and tongue muscles.

The anti-MuSK antibody assay should provide a valuable new diagnostic aid. Anti-MuSK antibodies may account not only for muscle weakness but also for the wasting that is occasionally seen in MG.

\section{Microneurographic recordings of C-nociceptive fibres in patients with erythromelalgia}

K Örstavik, R Schmidt, E Jörum, SM Chmelz, C Weidner, M Hilliges, E Torebjörk Department of Neurology, Ullevål Hospital, Oslo, Norway; Department of Clinical Neurophysiology, University Hospital, Uppsala, Sweden; Department of Neurology, Rikshospitalet, Oslo, Norway; Department of Physiology and Experimental Pathophysiology, University of Erlangen/Nurnberg, Germany; Department of Basic Oral Sciences, Karolinska Institute, Huddinge, Sweden

Microneurography studies on healthy subjects have shown that there are two subtypes of C-nociceptive fibres; mechanosensitive and mechanoinsensitive. These can be separated according to their transcutanous electrical thresholds and degree of slowing at low stimulus frequency. Induction of inflammation under experimental conditions has shown that mechanoinsensitive fibres can become sensitised to mechanical stimuli, probably due to reaction to chemical substances in inflammatory skin.

Erythromelalgia is a rare condition characterised by red, hot, painful extremities. The pathophysiological mechanism is thought to be shunting of blood through anastomoses in the skin, causing ischaemia in the superficial layers. Lately there have been reports on pathological changes in the peripheral nervous system in some of these patients. Preliminary results from an ongoing study in Oslo support this.

We have in the past year carried out microneurography studies on patients with erythromelalgia, looking for changes in single C-nociceptive fibres. The results are promising.

\section{The neurology of deep sea diving}

H Nyland, H Skeidsvold, E Thorsen Department, of Neurology, Clinical Neurophysiology, and Occupational Medicine, Haukeland Hospital, University of Bergen, Norway

Saturation diving on the North Sea oil fields is conducted in the depth range between 50 and 200 meters. A mixture of helium and oxygen is usually used as breathing gas. Diving exposure is characterised by its basic physical parameters; pressure, time, and gas mixture. Cumulative oxygen exposure, and decompression stress (inert gas accumulation and elimination) is calculated from these parameters. Diving to depths greater than $150 \mathrm{msw}$ may produce signs and symptoms of the high pressure nervous syndrome (HPNS); tremor, motor fatigue, impaired memory, dizziness, increased slow wave activity (2-7 Hz and depression of alpha waves in the EEG. Its severity increases with increasing depths. Decompression illness is caused by inert gas bubbles in the brain and spinal cord. The pattern of neurological injury varies widely, and the cerebral symptoms such as headache, fatigue and concentration difficulties may in some cases be mild and go unnoticed. Awareness of the possibility of long term neurological damage as a result of decompression illness or diving itself are subject to systematic surveys, and has been examined in neuropsychological, neurophysiological, and neuroimaging studies, which report mild to moderate changes in a proportion of the divers.

\section{Spinal cord decompression sickness: a personal review}

\section{Thrush Department of Neurology, Derriford Hospital, Plymouth, UK}

Diving is one of the fastest growing recreational sports in the United Kingdom. This has resulted from major developments in equipment, the introduction of PADI qualifications, and the plentiful supply of wrecks around the coast.

Like all sports it has its dangers and although uncommon, decompression sickness may cause devastating neurological damage. Each year in the United Kingdom there are about 12 deaths and 100 divers with serious decompression illness requiring recompression.

The Diving Diseases Research Centre opened in Fort Bovisand in 1983 and in 1996 relocated to the Tamar Science Park adjacent to Derriford Hospital. It offers a 24 hour advice and 24 hour emergency service and during the past 6 years it has recompressed on average 44 divers each year (36-52)

A retrospective review of 30 divers with spinal cord decompression sickness is presented. The most frequent initial symptoms were sensory disturbance (26), lower limb weakness (22), and bladder dysfunction (14). In 17 the first symptom was present on reaching the surface and in 13 the symptoms appeared within 30 minutes of surfacing. All patients were treated with intravenous fluids and hyperbaric oxygen and 14 received high dose corticosteroids. At discharge 14 patients had fully recovered, 16 had residual neurological sequelae.

The dispute as to whether paradoxical gas embolism is an important aetiological factor in spinal cord decompression illness will be discussed.

New developments of structural MRI using multiple sclerosis as a model for their application

DH Miller NMR Research Unit, Institute of Neurology, Queen Square, London, $U K$

While functional MR imaging has been of much interest in recent years in elucidating the anatomical basis of normal function, and its modification by disease, there has been a steady evolution of acquisition sequences and image analysis methods on MR scans, which have improved the quality of structural imaging in the CNS. Multiple sclerosis (MS) has been an interesting disease to evaluate such technique developments, as it includes a range of pathological changes involving focal lesions, normal appearing white matter, normal appearing grey matter, spinal cord, and optic nerves.

Traditional imaging techniques have included T2 weighted sequences to depict focal lesions, and gadolinium enhanced scanning to detect inflammation with a breakdown of the blood-brain barrier. More novel sequences in the past few years have included (1) MR spectroscopy to quantify metabolites, including $\mathrm{N}$-acetyl aspartate (a neuronal marker), and myoinositol (a possible glial marker); (2) magnetisation transfer imaging; (3) diffusion tensor imaging, and (4) sensitive automated methods for measuring tissue volumes in the brain, spinal cord, and optic nerves. Image analysis methods also allow segmentation of grey matter and white matter.

These methodological developments, all of which can be applied on standard 1.5 Tesla scanners, are briefly reviewed, using MS as a model to illustrate how such techniques can assist in understanding the natural history of the disease in monitoring therapeutic interventions. 


\section{Transcranial Doppler ultrasound}

D Russell Department of Neurology, The National Hospital, University of Oslo, Norway

Two recent advances in the use of transcranial Doppler (TCD) in stroke are described. The first is the introduction of multifrequency Doppler where the intracranial vessels are examined simultaneously using two different insonation frequencies $(2.0 \mathrm{MHz}$ and $2.5 \mathrm{MHz})$. This enables firstly the possibility of automatic detection of cerebral microemboli with the rejection of artifacts. Secondly, multifrequency Doppler can determine if the detected microembolus is composed of solid or gaseous elements. This is also possible with multifrequency Doppler as the amount of Doppler power being reflected back to the transducer at each insonation frequency is dependent on embolus type. Solid microemboli reflect more ultrasound at the higher frequency $(2.5 \mathrm{MHz}$ ) compared with the lower frequency $(2.0 \mathrm{MHz})$, whereas the opposite is the case for gaseous microemboli. This new possibility will enable us to better study the clinical significance of cerebral microembolus detection.

The second exciting observation is that ultrasound may promote thrombolysis. Several in vitro studies have shown that ultrasound has a significant thrombolytic effect which depends on exposure time, ultrasound intensity, and clot age. Preliminary clinical observations suggest that there is a higher recanalisation rate and better clinical recovery when TCD monitoring is carried out during tPA therapy.

\section{Predictive control in bimanual lifting and grasping: a} role for the cerebellum?

RM Bracewell, AM Wing University of Birmingham, Birmingham, UK

Background: When lifting an object with one hand, load force (LF) and grip force $(\mathrm{GF})$ are closely correlated. When conditions are stable, GF changes usually anticipate LF changes; this suggests predictive control mechanisms, thought to involve the cerebellum. Most everyday activities are, however, bimanual.

Aim: To investigate the role of the cerebellum in bimanual LF and GF coordination.

Methods: Lift task: the subject was required to lift and keep horizontal a platform held at either end by precision grip; the platform was loaded symmetrically or asymmetrically with different loads. Reflex task: the subject (eyes closed) had to hold the platform horizontally, while weights were dropped on it.

Subjects: 16 controls and one left handed patient with a left cerebellar lesion.

Results: Controls showed synchronous timing of left and right GF. In the lift task, the patient showed asynchronous GF timing (the ipsilesional hand lagging the right by $45 \mathrm{~ms}$ ). On the right, GF showed less anticipation of LF $(7 \mathrm{~ms})$ than controls $(15 \mathrm{~ms})$, and on the left GF lagged LF by $25 \mathrm{~ms}$.

However, in the reflex task, GF was synchronous ( $1 \mathrm{~ms}$ difference), but exhibited greater latency (about $125 \mathrm{~ms}$ ) than controls (about 85 $\mathrm{ms})$.

Conclusions: (1) The cerebellum may participate in predictive bimanual control.

(2) When one side is affected, the contralesional side may adjust.

Heart rate variability analysis in headache and muscle pain patients using the Wavelet transform

KB Nilsen, T Kristiansen, T Sand, M Gårseth, LJ Stovner, R Westgaard Department of Clinical Neurosciences, Norwegian University of Technology and Science, Trondheim, Norway

Different neurophysiological methods have been used in research of the autonomic nervous system in the past decades. The methods used for evaluating the hypothesised connection between the sympathetic nervous system and pain are many, and the results are not conclusive. Methods have been developed to evaluate both the function of the neuronal circuits and the degree of autonomic activation or arousal. In our laboratory we have tried to characterise the degree of sympathetic activation. We have tested patients with different pain complaints in a model developed in an occupational health research group.

Heart rate variability analysis has been used in research by others to characterise the level of sympathetic arousal. Different investigators have used different mathematical methods. Information about the variations in heart rate (frequency) can be extracted with different transformations of the original signal. We have compared the results obtained with the commonly used Fourier transform with the more complex Wavelet transform.
Our analysis shows that the methods are comparable when evaluating the frequency content of the heart rate signals, but the Wavelet transform has advantages when analysing the heart rate in a uninstructed or spontaneous setting.

How do we assess any benefits from interferon? Longitudinal data on 50 patients

MH Johnson, H Ford, S Denton Department of Neurology, St Fames's University Hospital, Leeds, UK

There are no established measures in use to monitor the progress of patients with multiple sclerosis (MS) on treatment with $\beta$-interferon. Apart from counting relapses, we cannot easily assess any benefits or the impact of side effects on these patients. In the Leeds MS treatment clinic, we have used the Leeds quality of life measure (LMSQOL) and the Guy's neurological disability scale (Guy's) at intervals before and during interferon treatment.

Results: Before treatment, the mean LMSQOL score was 23.7 (CI 22.1-25.5), the Guy's score was 12.1 (CI 10.3-13.9). The median EDSS score was 3.5 .

During the first year of treatment, there was a modest increase in quality of life: change in LMSQOL=2.9 (CI 1.5-4.3). This suggests a useful benefit, despite adverse effects which are common in the first few months of treatment. The Guys score did not change significantly. In the second year of treatment, there was no significant change in either measure. The EDSS score did not change.

In most patients, the quality of life and Guy's scores reflected changes with relapses or with progression. The questionnaires are easily administered by the MS nurse or doctor and could be used to monitor the benefits of disease modifying treatments in MS

\section{Extensive cortical demyelination in multiple sclerosis brains}

L Bø, H Nyland, C Vedeler, BD Trapp, S Mörk Department of Neurology and Pathology, Haukeland University Hospital, Bergen, Norway; Department of Neurosciences, Cleveland Clinic Foundation, OH, USA

Objectives: To examine the occurrence and extent of demyelination in the cerebral cortex in multiple sclerosis (MS) patients.

Methods: The material consisted of formalin fixed brains from 20 MS patients and six patients with no neurological disease. Irrespective of macroscopically evident lesions, tissue blocks were taken from the cingulate gyrus and frontal, temporal, and parietal lobes. Only specimens where strict sampling of brain tissue from the predetermined areas could be achieved were included. Paraffin embedded sections were immunostained for myelin basic protein. Areas of grey and white matter, and areas of demyelination were analyzed by morphometry.

Results: In control brains cortical demyelination was not detected. In 18 out of 20 MS brains there was cortical myelin loss. Median percentage demyelinated area in the cerebral cortex was $14.1 \%$ (mean: $26.5 \%$ ), in the subcortical white matter it was $0 \%$ (mean: $6.5 \%)$. The percentage demyelinated area was highest in the gyrus cinguli $(49.4 \%)$, lower in the temporal $(19.4 \%)$, frontal $(14.3 \%)$, and parietal lobe $(2.2 \%)$. Ninety two of 109 lesions, with $85.6 \%$ of the total cortical demyelinated area, were entirely intracortical. The most common pattern of cortical lesions was subpial demyelination, which was extensive in five $(25 \%)$ of the MS patients.

Conclusion: There is extensive demyelination in the cerebral cortex of MS patients. This may affect cortical functions and is of possible importance for the cognitive impairment often present in this disease.

\section{Epidemiology of multiple sclerosis: an overview}

R Midgard Department of Neurology, Molde Hospital, Molde, Norway

As a disease, multiple sclerosis (MS) is not evenly distributed with respect to either region, race, family, or individual. There is a striking difference in the frequency of MS between different parts of the world. Repeated epidemiological studies at regular intervals show an increase in prevalence rates. The worldwide distribution of MS can be seen as an indirect reflection of its cause, implicating environmental factors varying with latitude. There have been few population based studies of incidence of MS in a defined area over an extended period of time. When considering the surveys reporting MS incidence in the countries surrounding the North Sea, a decreasing trend is seen in Iceland, the Faroe Islands, the Orkney Islands, and Shetland, while Scotland, the United Kingdom, the Netherlands, Denmark, and the western coast of Norway show an increase which cannot entirely be attributed to alterations in survival or case ascertainment. 
Frequently asked questions from newly diagnosed MS patients are connected to prognostic factors for the development of disability and mortality, effects of pregnancy and oral contraceptives, and vaccination strategies. Recently published epidemiological studies focus on both descriptive and analytical epidemiological aspects of MS, and contribute to the understanding of these questions.

\section{Genetics of multiple sclerosis}

G Ebers University of Oxford, Oxford, UK

Multiple sclerosis is a complex trait determined by genes, environment, and very likely bad luck and epigenetic factors. The genetic epidemiology from the study of special groups gratatives is most consistent with an oligogenic hypothesis with major environmental factors which largely operate at a population level. It is not possible at this point to distinguish between a large number of genes with small effect versus a small number of genes with major effects and a large degree of heterogeneity. There may well be elements of both.

The search for specific genes has been disappointing to date. Few, if any, association studies outside of the MHC have reached any consensus. Multiple sclerosis investigators have been more conservative than those in type one diabetes where some 18 loci are now named, (IDDM 1-18) few of which have been replicated.

It is widely thought that the DRB allele 1501 specifically influences susceptibility to MS. However linkage disequilibrium within the MHC makes it difficult to be certain if this is the primary locus. There is evidence, however, which places the candidacy of this locus in more doubt than has been entertained. Specifically, the degree of haplotype sharing for MHC is (1) relatively low, (2) not different in sibling pairs lacking DRB 15, and (3) it seems to entail a hierarchy of susceptibility and resistance within the MHC. These findings have major implications for present day concepts of MS susceptibility.

It is clear that there must be non-MHC genes; however, genome searches have yet to highlight specific areas which have reached consensus. In some complex disorders the way forward has been with the identification of mendelian families. Although these may constitute only a small percentage of the total number of familial cases, they would have the advantage of permitting entry into a pathway which would identify potential disease mechanisms. Results from a search for mendelian families seen during the Canadian collaborative study are presented.

\section{Multiple sclerosis In Troms and Finnmark}

M Kampmann Department of Neurology, Regional Hospital, University of Tromsø, Norway

The two northernmost counties of Norway, Troms and Finnmark, have a population of about 225000 , about $10 \%$ of which belong to the indigenous Sami people. The epidemiology of multiple sclerosis (MS) has been studied in Troms and Finnmark since 1954. Until 1983 this region had the lowest incidence of MS of any area in Scandinavia. However, the mean annual incidence rate has increased from 1.8 per 100000 during $1954-73$ to 3.5 per 100000 during the period 1983-92, and prevalence increased from 20.6 per 100000 in 1973 to 73.0 per 100000 in 1993. During the 1990s MS has been diagnosed among the Sami population for the first time.

The entire population of Troms and Finnmark is served by the Department of Neurology of the Tromsø University hospital. About $20 \%$ of the patients are treated with interferon- $\beta$ in our MS clinic. These patients are monitored with scoring on the expanded disability status scale (EDSS) and assessment of the MS functional composite (MSFC).

\section{Clustering of multiple sclerosis cases in Tayside and their relation with deprivation and population density}

JDE Parratt, S Wilson, P Donnan, J O'riordan, S Swingler Department of Neurology, Tayside University Hospitals Trust, UK

Clusters of multiple sclerosis (MS) have previously been identified and used as evidence for either genetic or more commonly environmental hypotheses regarding the aetiology of the disease. Such studies have been criticised, however, due to difficulty in defining the absolute onset of the disease and tenuous methodology. We identified clustering of MS cases in Tayside, Scotland using a robust regression model and examined clusters for associations with deprivation and population density.

On the prevalence date of 1 December 2000, 998 cases of clinically definite or probable MS were registered in Tayside (population 378 367) yielding a crude prevalence of 302 per 100000 ( $95 \%$ CI
263-340). Cluster analysis revealed a rural area consisting of four postcode sectors with significantly higher prevalence than the rest of the region (expected cases 37, observed cases 60, $\mathrm{p}=0.027$ ). Deprivation (DS) status in this area (mean DS 2.75, 95\% CI 1.95-3.55) was lower than the rest of Tayside (mean DS 3.38, 95\% CI 3.01-3.75) and population density was also low. Correlation of MS prevalence with deprivation and population density in all 68 postcode sectors of the region revealed inverse relations (deprivation: Pearson correlation coefficient $-0.23, \mathrm{p}=0.06$; population density $-0.287, \mathrm{p}=0.017$ ).

This study has not taken account of the place of residence of each patient when they most likely developed the disease-namely, adolescence - but rather at clinical onset. Despite this, we have demonstrated a spatial cluster of MS in a relatively static population. This area is associated with lower deprivation and population density and therefore is consistent with Alter's theory that late life exposures to common infections result in immune sensitisation and a population propensity towards MS.

\section{Search for susceptibilty genes in multiple sclerosis}

HF Harbo, EG Celius, K-Z Dai, F Vartdal, B Vandvik, A Spurkland Institute of Immunology, The National Hospital, University of Oslo; Department of Neurology, Ullevål Hospital, University of Oslo, Norway

Methods: The aim of our project is to contribute to the search for genes that are associated with MS. We have used the candidate gene strategy typing about 300 Norwegian MS patients and 300 controls and 177 Nordic sib pair families focusing on the CTLA4 gene (cytotoxic T lymphocyte antigen 4 ), the $S H 2 D 2 A$ gene (encoding $\mathrm{T}$ cell specific adapter protein) and the $H L A$ (human leucocyte antigen) complex. Furthermore we participate in the European genome wide linkage disequilibrium collaborative project GAMES (genetic analysis of multiple sclerosis in Europeans). We type pooled samples from 199 Scandinavian patients with familiar MS and 200 unrelated controls for 6000 markers positioned at about every $0.5 \mathrm{CM}$ all over the genome.

Results: Our studies show that the CTLA4 +49 A/G genotype is associated with MS $\left(\mathrm{p}_{\mathrm{c}}=0.01\right)$, and that MS is associated with short alleles in the promoter of the $S H 2 D 2 A$ gene $\left(\mathrm{p}_{\mathrm{c}}=0.03\right)$. These data suggest that genetic susceptibility to MS is conferred in part via regulatory control of T cell activation. Further results show that female sex $(p=0.0251)$ and young age at diagnosis $(p=0.0254)$ are correlated with the HLA-DR, DQ6 haplotype in MS. Our contribution to the ongoing GAMES study in Cambridge, UK is discussed.

Relation between serial MRI and the development of disability in patients with clinically isolated syndromes suggestive of multiple sclerosis

PA Brex, O Ciccarelli, JI O'Riordan, M Sailer, AJ Thompson, DH Miller NMR Research Unit, Institute of Neurology, University College London, UK

Introduction: The presence of asymptomatic brain lesions on T2 weighted MRI of patients presenting with clinically isolated syndromes (CIS) increases the risk of multiple sclerosis(MS) over the next 10 years. The relatively short duration of most studies has meant that little information has been obtained on the relation between early MRI findings and long term disability.

Methods: Serial MRI and clinical examinations were performed on 71 CIS patients over a mean of 14.1 years. Disability was measured on the expanded disability status scale (EDSS).

Results: Clinically definite MS developed in 44/50 (88\%) with an abnormal MRI at presentation, compared with $4 / 21$ (19\%) with a normal MRI. The median EDSS of the MS patients was 3.25 (range 0 B 10 ); $31 \%$ had an EDSS $\geqslant 6$ (including three deaths due to MS). EDSS at 14 years was found to correlate more strongly with the increase in T2 lesion volume over the first 5 years $(r=0.61)$ than with baseline T2 lesion load $(r=0.48)$.

Conclusions: Early T2 lesion volume increase was a better predictor of long term disability than T2 lesion volume at presentation, suggesting that the early pathological course has an important influence on the long term clinical outcome.

\section{Organisation of stroke services}

CP Warlow Department of Clinical Neurosciences, University of Edinburgh, Edinburgh, UK

Ideally, the care of any group of patients should be determined by their needs, and on the basis of evidence that the benefits of any therapeutic interventions are greater than the risks, and that the costs 
are affordable. For stroke, unlike many or perhaps even most other disorders, we have a very good idea of incidence, case fatality, residual disability, prevalence, and hospital admission rates which make for a good start in designing stroke services. Also, for interventions, we know that for acute stroke early admission to a stroke unit rather than a chaotic general medical ward, aspirin, and maybe thrombolysis for a few, are all well worthwhile. For secondary prevention we know that aspirin, the control of vascular risk factors, and carotid surgery for a few are also worthwhile, and that any interventions must be quick because of the high early recurrence rate. We have far less information about expensive interventions such as nursing care and various forms of therapy. It follows, therefore, that stroke services need to be organised, and that requires a leader, usually a doctor, and in the United Kingdom usually a geriatrician and ideally in partnership with a neurologist. 24 hour a day availability of brain imaging is crucial, and also collaboration with neurosurgeons, vascular surgeons, and cardiologists at least. Stroke services also have to react quickly, for two reasons. Firstly, to provide thrombolysis in acute stroke, and secondly to initiate secondary prevention within a few days. Any stroke service must also provide education and training for all who work in it and for others, support for carers, a resource for stoke information for the surrounding community, and also opportunity for clinical research. Auditing of stroke services is problematical, but keeping an eye on process rather than outcome is perhaps the best way to go-for example, the proportion of patients getting a CT scan within 24 hours, the proportion of ischaemic stroke survivors and patients with transient ischaemic attack discharged on aspirin, and so on. It would be nice to think that such perfect stroke services were widespread in the United Kingdom, but unfortunately that is not the case (yet).

\section{Acute stroke: the first 6 hours}

L Thomassen, U Waje-Andreassen, C Maintz Department of Neurology, Haukeland Hospital, University of Bergen, Norway

The goals of intervention in acute stroke are to dissolve the occluding clot and restore normal blood flow (thrombolysis) and to protect the brain by interrupting or slowing the ischaemic cascade (neuroprotection). Time is crucial.

Our data indicate that information to the public and improved admission routines reduce admission delay, whereas patient behaviour does not change easily.

Existing data indicate that the benefit of intravenous thrombolytic therapy within 3 hours after stroke onset clearly outweigh the risks. Among 24 patients treated in our department, 10 (42\%) demonstrated early clinical improvement compatible with a positive thrombolysis effect. Three patients died, none had a symptomatic haemorrhage. At follow up after 6 months, 10 patients $(42 \%)$ had achieved independence (modified Rankin scale: mRS 0-2), 9 (33\%) had an unfavourable outcome (mRS 3-5), and five patients $(21 \%)$ had died.

Stroke is a heterogeneous entity and early diagnostic testing should establish regional blood flow and anatomical regions irreversibly damaged or at risk. In our patients, Doppler ultrasound examination at admission demonstrated highly heterogenous flow patterns in clinically similar patients, where rapid recanalisation was related to rapid clinical improvement. Immediate Doppler ultrasonography increases our understanding of the vascular pathology to be treated and supports a pathophysiologically driven stroke therapy.

"No consent, please-we're British!” Will observational research in neurology survive changes in data protection law and confidentiality guidance?

R Al Shahi, CP Warlow Department of Clinical Neurosciences, University of Edinburgh, Western General Hospital, Edinburgh, UK

Hitherto, there has been an almost unique opportunity in countries like Britain and Norway for high quality, unbiased observational research.

Across the world rapid changes in data protection law (for example, the British Data Protection Act (1998) and the Norwegian Personal Data Act (2000)), technology and society are reshaping the way identifiable information about patients is handled. There is a real risk that these changes could seriously jeopardise audit, clinical governance, and observational epidemiological research, thereby compromising patient care and the public interest.

Ideally, of course, patients in a research study or audit should have given either explicit or implicit consent to the use of data that preferably should not identify them directly. Systematic bias could well invalidate the findings of observational studies if people were excluded for lack of their consent. This would not just hinder the progress of medical knowledge, but might well lead to completely incorrect conclusions, so damaging the public interest.

Further legal clarification, consistent guidance from professional organisations, and unbiased public consultation are required. What is the ideal balance between individual confidentiality and data protection on the one hand, and on the other the legitimate use in the public interest of patient identifiable data without consent?

\section{Epilepsy in elderly people after stroke}

OM Rønning, M Lossius Department of Neurology, Central Hospital of Akershus, University of Oslo, Norway

Objectives: Seizure after stroke is held as one of the major causes of epilepsy in elderly people. This prospective study was performed to determine the outcome of patients $\geqslant 60$ years old with acute stroke admitted to a hospital including registration of medical complications as seizures.

Methods: A consecutive cohort of 478 patients admitted to a single hospital due to acute stroke and who survived the first 2 weeks. Follow up was 5 years. The main outcome measures were occurrence of a single seizure after stroke and recurrent seizures.

Results: Epilepsy occurred in $21(4.4 \%)$ of 478 patients with stroke. All of these patients had a cerebral infarction. Compared with patients without epilepsy after stroke patients with recurrent seizures had a significantly worse initial neurological score (median Scandinavian stroke scale score 30 in the epilepsy group and 40 in patients without epilepsy; $p=0.014$ ) and was more dependent in Barthel's activities of daily living $(7 / 20$ in the epilepsy group and $11 / 20$ in the non-epilepsy group; $\mathrm{p}=0.004$ ).

Conclusions: Epilepsy after stroke occurs more often in patients with severe ischaemic strokes. We could not confirm a higher incidence of recurrent seizures among patients with haemorrhagic strokes.

\section{Evidence based guidelines for the management of narcolepsy}

A Zeman for the Narcolepsy Management Guidelines Working Party Department of Clinical Neurosciences, University of Edinburgh, Western General Hospital, Edinburgh, UK

Narcolepsy is a disorder of the sleep-wake cycle, characterised by excessive daytime sleepiness, cataplexy, sleep paralysis, hypnagogic hallucinations, and disruption of nocturnal sleep. Many of its features can be understood in terms of the intrusion of elements of rapid eye movement sleep into the waking state. Onset can occur at any age, most often in the second and third decades. The disorder is moderately common, with a prevalence in Europe of circa 4/10 000 . Diagnosis is often delayed. Pharmacological treatment is effective for sleep attacks and cataplexy; attention to associated psychosocial problems is also important.

We have established a multidisciplinary working party to draw up management guidelines based on review of all available evidence. The issues addressed include: the predictive value of the symptoms of narcolepsy and their differential diagnosis; the optimal pathway for investigation, including HLA typing, polysomnography and multiple sleep latency testing; the medical treatment of narcoleptic sleepiness, cataplexy and sleep disturbance; the management of associated psychological and social difficulties; and the implications of our guidelines for service provision.

The guidelines are in their first draft and will be nearing their final form in May. We value an opportunity to present them to the ABN as work in progress.

Animal models in the study of antiepileptic drugs and reproductive endocrine function

L Sveberg Røste, E Taubøll, A Krogenæs, J Isojärvi, L Gjerstad Department of Neurology, Rikshospitalet, University of Oslo, Norway; The Norwegian School of Veterinary Science, Oslo, Norway; Department of Neurology, University of Oulo, Finland

Reproductive endocrine disorders are often found in patients with epilepsy. This has been related both to the epilepsy itself, but also to the use of antiepileptic drugs, especially valproate (VPA).

The effects of VPA on endocrine function and gonadal morphology was studied in a series of animal experiments. Firstly, female Wistar rats were fed perorally with VPA $(400 \mathrm{mg} / \mathrm{kg} / \mathrm{day}(\mathrm{n}=15)$ or 600 
$\mathrm{mg} / \mathrm{kg} /$ day $(\mathrm{n}=20))$, lamotrigine $(10 \mathrm{mg} / \mathrm{kg} / \mathrm{da}) \mathrm{y}$, or control solution $(n=15)$ for 90 days. There was a significant, dose dependent increase in the number of follicular ovarian cysts and a reduction in the number of corpora lutea after VPA but not lamotrigine treatment. A direct effect on steroidogenesis was demonstrated in an additional study utilising cultures of pig ovarian follicles. In male Wistar rats fed with VPA $(400 \mathrm{mg} / \mathrm{kg} /$ day $(\mathrm{n}=15), 800 \mathrm{mg} / \mathrm{kg} /$ day $(\mathrm{n}=20))$, or control solution $(n=15)$ for 90 days there was a significant reduction in testicular weight in the high dose treated VPA group. 15/20 of these animals showed widespread testicular atrophy and reduced spermatogenesis. High dose VPA treated animals also showed a significant rise in $\mathrm{LH}$ and FSH with reduced free testosterone and unchanged total testosterone. In a new study, male goats fed perorally with VPA for 8 months with serum concentrations in the lower therapeutic range showed a reduction in testicular size.

In conclusion, our studies have shown a drug specific effect of VPA on endocrine function and gonadal morphology in non-epileptic female and male animals. The precise mecahanisms of action remain to be elucidated. The clinical relevance of these findings needs consideration.

\section{Clinical neurology in Norway: past, present, and future}

E Dietrichs Department of Neurology, Ullevål Hospital, University of Oslo, Norway

Skulls with trepanation holes prove that diseases in the CNS were diagnosed and treated in Norway more than 5000 years ago. However, written documentation only dates back to the Viking age. After the battle of Hlyrskog Heath in AD 1043, King Magnus the Good picked Atle, father of Bård Svarte from Selårdal on Iceland, for treating the wounded. Atle became the ancestor of a famous family of doctors. His great grandson Hrafn Sveinbjarnarson may have been trained in Salerno. According to his own saga, he treated seizures by cauterisation at certain points on the head.

Early Norwegian neuroscience counts names as GH Armauer Hansen (1841-1912), the discoverer of Mycobacterium leprae, and his father in law, D C Danielsen (1815-94). They mapped the neurological manifestations of leprosy (Hansen's disease). Fridtjof Nansen (18611930), best known as an Arctic explorer and Nobel Peace Prize laureate, was the first scientist to give strong evidence for the "neuron theory". Later important contributors to basic neurosciences are the members of "the Oslo school of neuroanatomy" - for example, Jan B Jansen (1898-1984) and Alf Brodal (1910-88)

However, Norwegian clinical neurology has a very distinctive "electrical root". Chr Heiberg (1799-1872), the professor of surgery at the University of Oslo Medical School, used "Remak's electrotherapy" so extensively that the National Hospital had to employ an "electrotherapeut". Different doctors served in this position from 1858 to 1885 . That year Chr Blom Leegaard (1851-1921) was asked to take up this work. Leegaard was trained as a clinical neurologist and neuropathologist in Germany, Austria, and France. He accepted the position only if "the electrotherapist is given the duty to lecture and train medical students in diseases of the nervous system and electrotherapy". Leegaard was granted this. In 1893 he was appointed associate professor of neurology, in 1895 full professor. The first department of neurology, also at the National Hospital, was founded in the same year. Leegaard was succeeded as professor of neurology first by G H Monrad Krohn (1884-1964), world famous for his book Clinical examination of the nervous system, and then by Sigvald Refsum (1907-91), known for the disease bearing his name (phytanic acid storage disease). Refsum was also elected president of the World Federation of Neurology.

After the second world war new neurological departments were opened at Ullevål hospital in Oslo (1948), Haukeland hospital in Bergen (1952), St. Joseph's hospital in Porsgrunn (1954), and at Drammen hospital (1955). By 2001 neurology departments are found in 23 hospitals; in all counties except Finnmark. These departments take care of patients with various neurological diseases. However, regional differences exist, and whereas acute neurological conditions such as stroke and sciatica in many counties are treated in the neurological departments, this is not the case in all hospitals. Similarly, dementia and neurorehabilitation in many counties are handled by other specialties.
The neurological departments of the university hospitals are active in various fields-for example, muscular diseases (Tromsø), headaches (Trondheim), neurosonology (National hospital), and neuroimmunology (Haukeland, Ullevål). Several of the county hospitals are also active in research (for example, the Parkinson project in Stavanger).

Among a population of about 4.5 million people, Norway has about 600 hospital beds for patients with neurological diseases. We have about 200 specialists in clinical neurology, most of them working in hospitals. The demand for neurological services has shown a steady increase. Due to the rapid progress of knowledge in the neurosciences and new diagnostic and therapeutic possibilities in clinical neurology, an increasing number of neurologists and hospital beds will be required. The Norwegian Neurological Association claims that all diseases of the nervous system, including stroke, sciatica and dementia, as well as neurorehabilitation, should be handled by, or in close collaboration with, specialists in clinical neurology.

\section{Neurology in the United Kingdom}

D Stevens Cheltenham, UK

Neurological services in the United Kingdom have developed slowly, many think too slowly, and the distribution of neurologists and the facilities that they require to work effectively have evolved in a strange and patchy manner. While the reasons for this curious pattern of evolution is of interest, our plans for the future are of much greater importance. The Association of British Neurologists has already produced a template for a truly equitable service for the whole country and this is being further refined at the moment. We recognise, however, that there are many reasons why the implementation of these plans may prove to be difficult. A discussion of these issues could be of interest to our Norwegian colleagues, for they may learn from our experiences. However, we think it quite possible that we will have much to learn from their experience in creating a national neurological service in a country which is geographically more challenging than the United Kingdom.

\section{Handling of neurology referrals to a DGH clinic: impact of GP clinical assistants}

DF Smith, M Vlies, R Bartolo, JP Leach Wrexham Maelor Hospital, Wrexham; The Walton Centre for Neurology and Neurosurgery, Liverpool, UK

Neurology outpatient waiting times are unacceptable. Recommendations for expansion of neurology consultant grade (1:100 000) will not be achieved until 2019. Alternative models of care must be developed and tested.

Suspected epilepsy is a common reason for referral. This project assessed the impact of a GP clinical assistant on the waiting times for possible new epilepsy and all other referrals.

Data collection included date and reason for referral, date seen and diagnosis, of patients seen in the year before (1999) and the year after (2000) intervention. This involved (a) 4 months training for appointed GP (b) additional new patient slots specifically for suspected new epilepsy, and (c) two GP sessions per month.

The $30 \%(n=66)$ increase in patients seen was attributable to newly created appointments. The median waiting time for patients with suspected new epilepsy referred between January and June 99, July and December 99, January and June 2000, and July and December 2000 were $125,101,37$, and 43 days. The proportions of these patients seen within 90 days were $33.3 \%, 42.1 \%, 96.8 \%$, and $95.4 \%$. The corresponding median waiting times for other referrals were 164, 153, 116.5 , and 57.5 days while the proportions seen within 90 days were $24.7 \%, 25.5 \%, 39 \%$, and $73.5 \%$.

The model described represents a feasible method of managing neurology waiting lists. A logical extension is the creation of intermediate specialists linking primary and secondary care. Their impact on neurology waiting times could be tested in a prospective randomised study. 